\title{
COMPOSITION SERIES AND INTERTWINING OPERATORS FOR THE SPHERICAL PRINCIPAL SERIES
}

\author{
BY KENNETH JOHNSON AND NOLAN R. WALLACH ${ }^{1}$
}

Communicated by I. M. Singer, April 13, 1972

1. Introduction. The purpose of this note is to announce several results (to appear in [4] and [5]) on the spherical principal series for a semisimple Lie group $G$. Our main result gives a complete determination of the composition series for the case when $G$ has split rank 1 . We also give explicit formulas for the intertwining operators for the spherical principal series (for arbitrary rank) and thereby have explicit formulas for parts of the corresponding generalized $c$-functions. Using the intertwining operators we determine which paris of the composition series of a spherical principal series representation are unitarizable in the case when $G$ has split rank 1 .

Except for $\S 5$, we exclude the case where $G=S L(2, R)$ from the statement of our theorems. For the analogous discussions for $G=S L(2, \boldsymbol{R})$ see Gelfand, Graev, Vilenkin [1] or Sally [9].

2. Notation and spherical harmonics. Let $G$ be a real, connected, semisimple, Lie group with finite center. Let $G=K A N$ be an Iwasawa decomposition of $G$ and let $M$ and $M^{\prime}$ denote respectively the centralizer and normalizer of $A$ in $K$. Then $W=M^{\prime} / M$ is the Weyl group of $G / K$. Let $\mathfrak{g}, \mathfrak{f}, \mathfrak{a}, \mathfrak{m}$, and $\mathfrak{n}$ be respectively the Lie algebras of $G, K, A, M$ and $N$. If $g \in G$ then $g=k(g) \exp (H(g)) n(g)$ where $k(g) \in K, H(g) \in \mathfrak{a}$ and $n(g) \in N$ and $k(g), H(g)$ and $n(g)$ are unique.

Let $\mathfrak{a}^{*}$ be the dual of $\mathfrak{a}$ and $\mathfrak{a}_{c}^{*}$ its complexification. If $\gamma \in \mathfrak{a}^{*}-\{0\}$ then $\gamma$ is called a restricted root if $g_{\gamma}=\{X \in \mathfrak{g} \mid[H X]=\gamma(H) X$ for all $H \in \mathfrak{a}\}$ is not $\{0\}$. Let $m_{\gamma}=\operatorname{dim} \mathfrak{g}_{\gamma}$ and let $\Delta$ denote the set of restricted roots. Then $\mathfrak{g}=\sum_{\gamma \in \Delta} \mathfrak{g}_{\gamma}+\mathfrak{m}+\mathfrak{a}$. We have an ordering on $\Delta$ such that if $\Delta^{+}$is the set of positive roots then $\mathfrak{n}=\sum_{\alpha \in \Delta^{+}} \mathfrak{g}_{\alpha}$. Let $\bar{N}$ be the Lie group corresponding to $\overline{\mathrm{n}}=\sum_{\alpha \in \Delta^{+}} \mathfrak{g}_{-\alpha}$. Let $2 \rho=\sum_{\alpha \in \Delta} \mathfrak{g} m_{\alpha} \alpha$.

If $\lambda \in \mathfrak{a}_{C}^{*}$ we can define a character on $M A N$ by sending $x=$ man $\rightarrow x^{\lambda}$ $=e^{\lambda(\log a)}$. On $B=G / M A N=K / M$, we fix the unique $K$-invariant measure $d b$ such that $\int_{B} d b=1$.

Let $H^{\lambda}$ be the set of all measurable functions $f: G \rightarrow C$ such that

(1) $f(g x)=x^{-\lambda} f(g)$ if $x \in M A N$; and,

(2) $\int_{B}|f(b)|^{2} d b<\infty$.

AMS 1970 subject classifications. Primary 22E45, 22D30; Secondary 22E43.

${ }^{1}$ Alfred P. Sloan Fellow at Rutgers University. 
Let $\pi_{\lambda}$ denote the action of $G$ on $H^{\lambda}$ induced by left translation. If $X^{\lambda}$ is the space of $K$-finite elements of $H^{\lambda}$ then the elements of $X^{\lambda}$ are actually analytic functions on $G$. $\pi_{\lambda}$ thus induces an action of $U(\mathrm{~g})$ (the complexified universal enveloping algebra of $\mathfrak{g}$ ) on $X^{\lambda}$.

Let $\widehat{K}$ denote the set of equivalence classes of irreducible finite-dimensional representations of $K$, and if $\pi: K \rightarrow G L(V)$ is a representation of $K$ let $V^{M}=\{v \in V \mid \pi(m) v=v$ for all $m \in M\}$. Fix, for $\gamma \in \hat{K},\left(\pi_{\gamma}, V_{\gamma}\right) \in \gamma$. Let $\hat{K}_{0}=\left\{\gamma \in \hat{K} \mid V_{\gamma}^{M} \neq(0)\right\}$.

Suppose $\operatorname{dim} \mathfrak{a}=1$. Then $\mathfrak{g}=\mathfrak{g}_{-2 \alpha} \oplus \mathfrak{g}_{-\alpha} \oplus \mathfrak{m} \oplus \mathfrak{a} \oplus \mathfrak{g}_{\alpha} \oplus \mathfrak{g}_{2 \alpha}$ where $m_{2 \alpha}$ is either $0,1,3$ or 7 . We assume that $\mathfrak{n}=g_{\alpha} \oplus g_{2 \alpha}$. Then $2 \rho=\left(m_{\alpha}+2 m_{2 \alpha}\right) \alpha$.

THEOREM 2.1 (KOSTANT [7]). As a representation of $K$,

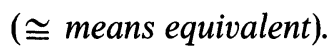

$$
X^{0} \cong X^{\lambda} \cong \sum_{\gamma \in K_{0}} V_{\gamma}
$$

In particular, each irreducible subrepresentation of $K$ occurs exactly once.

There is a natural parametrization of $\widehat{K}_{0}$ for each $G$ which is given as follows:

(1) If $m_{2 \alpha}=0, \hat{K}_{0}$ may be identified with the nonnegative integers.

(2) If $m_{2 \alpha}=1, \hat{K}_{0}$ may be identified with the set $\{(m, k) \mid k$ and $m$ are integers, $|k| \leqq m$ and $m-k$ is even $\}$.

(3) If $m_{2 \alpha}=3$ or $7, \hat{K}_{0}$ may be identified with the set $\{(m, k) \mid k$ and $m$ are nonnegative integers and $m-k$ is even $\}$.

We will identify $\widehat{R}_{0}$ with the sets defined in (1), (2), (3).

If $\lambda \in \mathfrak{a}_{C}^{*}, \lambda=\lambda_{1} \alpha$ where $\lambda_{1} \in C$. We abuse notation and set $\lambda_{1}=\lambda$. Let $H \in \mathfrak{a}$ such that $\alpha(H)=1$.

THEOREM 2.2. We may select a basis $\left\{e_{\gamma} \mid \gamma \in \widehat{K}_{0}\right\}$ of $\left(X^{0}\right)^{M}$ such that

(1) If $m_{2 \alpha}=0$,

$$
\begin{aligned}
\pi_{\lambda}(H) e_{m}=\frac{1}{m_{\alpha}+2 m-1}\left[\left(m_{\alpha}+\right.\right. & m-1)(\lambda+m) e_{m+1} \\
& \left.+m\left(\lambda+1-m-m_{\alpha}\right) e_{m-1}\right] .
\end{aligned}
$$

(2) If $m_{2 \alpha}=1$,

$$
\begin{aligned}
\pi_{\lambda}(H) e_{m, k}=\frac{1}{2\left(m_{\alpha}+2 m\right)}[ & \left(m_{\alpha}+k+m\right)(\lambda+k+m) e_{m+1, k+1} \\
& +(m-k)\left(\lambda-m+k-m_{\alpha}\right) e_{m-1, k+1} \\
& +\left(m-k+m_{\alpha}\right)(m-k+\lambda) e_{m+1, k-1} \\
& \left.\quad+(k+m)\left(\lambda-m_{\alpha}-m-k\right) e_{m-1, k-1}\right] .
\end{aligned}
$$


(3) If $m_{2 \alpha}=3$,

$\pi_{\lambda}(H) e_{m, k}$

$$
\begin{aligned}
=\frac{1}{2\left(m_{\alpha}+2+2 m\right)}[ & \left(m_{\alpha}+2+k+m\right)(\lambda+k+m) e_{m+1, k+1} \\
& +(m-k)\left(\lambda-m+k-m_{\alpha}-2\right) e_{m-1, k+1} \\
& +\left(m_{\alpha}+m-k\right)(m-k-2+\lambda) e_{m+1, k-1} \\
& \left.+(k+m+2)\left(\lambda-m-k-m_{\alpha}-4\right) e_{m-1, k-1}\right] .
\end{aligned}
$$

(4) If $m_{2 \alpha}=7$, then $m_{\alpha}=8$ and

$$
\begin{aligned}
\pi_{\lambda}(H) e_{m, k}=\frac{1}{2(k+3)(14+2 m)}[ & (k+6)(\lambda+m+k)(14+m+k) e_{m+1, k+1} \\
& +(k+6)(\lambda-m+k-14)(m-k) e_{m-1, k+1} \\
& +k(\lambda+m-k-6)(8+m-k) e_{m+1, k-1} \\
& \left.+k(\lambda-20-m-k)(m+k+6) e_{m-1, k-1}\right] .
\end{aligned}
$$

The proof of Theorem 2.2(1), (2), (3) will appear in [5] and that of Theorem 2.2(4) will appear in [4].

3. Intertwining operators and generalized $c$-functions in the rank 1 case. Again let $\operatorname{dim} \mathfrak{a}=1$, and let $w \in M^{\prime}$ such that $A d w=-1$ on $\mathfrak{a}$.

If $\gamma \in \hat{K}_{0}, \tau \in \gamma$ and $e_{\gamma} \in\left(X_{\gamma}^{0}\right)^{M}$ we now compute

$$
\tau(w) \int_{\bar{N}} \tau(k(\bar{n})) e_{\gamma} e^{-\lambda(H(\bar{n}))} d \bar{n}=c_{\alpha}(\lambda, \gamma) e_{\gamma}
$$

or more precisely its meromorphic continuation (here we normalize the Haar measure on $\bar{N}$, $d \bar{n}$, so that $\left.\int_{\bar{N}} e^{-2 \rho(H(\bar{n}))} d \bar{n}=1\right)$. Recall from Helgason [3] that

$$
\int_{\bar{N}} e^{-\lambda(H(\bar{n}))} d \bar{n}=c(i(\rho-\lambda))=\frac{c_{0} 2^{\rho-\lambda} \Gamma(\lambda-\rho)}{\Gamma\left(\frac{1}{2}\left(\lambda-m_{2 \alpha}+1\right)\right) \Gamma\left(\frac{1}{2} \lambda\right)},
$$

where $c_{0}=\Gamma\left(\frac{1}{2}\left(m_{\alpha}+m_{2 \alpha}+1\right)\right) 2^{\left(m_{\alpha}+2 m_{2 \alpha}\right) / 2}$.

We can now state our results.

THEOREM 3.1. (1) If $m_{2 \alpha}=0$,

$$
c_{\alpha}(\lambda, m)=c(i(\rho-\lambda)) \prod_{j=1}^{m}\left(\frac{m_{\alpha}-1-\lambda+j}{\lambda+j-1}\right) .
$$

(2) If $m_{2 \alpha}=1$, 


$$
c_{\alpha}(\lambda,(m, k))=c(i(\rho-\lambda)) \prod_{j=1}^{(m-k) / 2}\left(\frac{m_{\alpha}+2 j-\lambda}{2 j-2+\lambda}\right) \prod_{j=1}^{(m+k) / 2}\left(\frac{m_{\alpha}+2 j-\lambda}{2 j-2+\lambda}\right) .
$$

(3) If $m_{2 \alpha}=3$,

$$
c_{\alpha}(\lambda,(m, k))=c(i(\rho-\lambda)) \prod_{j=1}^{(m-k) / 2}\left(\frac{\lambda-m_{\alpha}-2-2 j}{4-2 j-\lambda}\right) \prod_{j=1}^{(m+k) / 2}\left(\frac{2 j+m_{\alpha}+4-\lambda}{2 j-2+\lambda}\right) .
$$

(4) If $m_{2 \alpha}=7$,

$$
c_{\alpha}(\lambda,(m, k))=c(i(\rho-\lambda)) \prod_{j=1}^{(m-k) / 2}\left(\frac{\lambda-2 j-14}{8-2 j-\lambda}\right) \prod_{j=1}^{(m+k) / 2}\left(\frac{20+2 j-\lambda}{2 j-2+\lambda}\right) .
$$

The proofs of Theorem 3.1(1), (2), (3) will appear in [5]; that of Theorem 3.1(4) will appear in [4].

Let $d(\lambda)=2^{\rho-\lambda} \Gamma(\lambda-\rho)$ and $e(\lambda)^{-1}=\Gamma\left(\frac{1}{2}\left(\lambda-m_{2 \alpha}+1\right)\right) \Gamma\left(\frac{1}{2} \lambda\right)$. Let $a_{\alpha}(\lambda, \gamma)=c_{\alpha}(\lambda, \gamma) / d(\lambda)$. Note that $a_{\alpha}(\lambda, \gamma)$ is an analytic function of $\lambda$.

REMARK. The case $m_{\alpha}=1$ and $m_{2 \alpha}=0$ has been done by Gelfand et al. [1] and Sally [9]. Here $\hat{K}_{0}$ is identified with all integers and

$$
c_{\alpha}(\lambda, n)=c(i(\lambda-\rho)) \prod_{j=1}^{|n|} \frac{j-\lambda}{\lambda+j-1} .
$$

Now define $a_{\alpha}(\lambda, n)$ as above.

Let $A_{\lambda}: X^{\lambda} \rightarrow X^{2 \rho-\lambda}$ be the linear operator such that $\left.A_{\lambda}\right|_{X_{\gamma}^{\lambda}}=a_{\alpha}(\lambda, \gamma) I$.

THEOREM 3.2. Suppose $B: X^{\lambda} \rightarrow X^{v}$ is a nonzero operator which intertwines $\pi_{\lambda}$ and $\pi_{v}$. Then either $v=\lambda$ or $v=2 \rho-\lambda$. Moreover, if $v=\lambda, B$ is a scalar multiple of the identity; and if $v=2 \rho-\lambda, B$ is a scalar multiple of the operator $A_{\lambda}$.

Theorem 3.2 is a consequence of Theorem 2.2. Actually using results of Schiffmann [10], Theorem 3.1 follows from Theorem 3.2.

If $f, g \in X^{\lambda}$, let $(f, g)=\int_{B} f(b) \overline{g(b)} d b$ and let $(f, g)_{\lambda}=\left(A_{\lambda} f, g\right)$. Letting $\pi_{\lambda}(U(\mathrm{~g})) 1_{0}=X_{0}^{\lambda}$ we have

THEOREM 3.3 (KosTANT [7]) (1). If $m_{2 \alpha}=0$ and $\lambda$ is real and $0 \leqq \lambda<m_{\alpha}$, $(,)_{\lambda}$ defines a positive definite Hermitian inner product on $X_{0}^{\lambda}$. Thus by $N$ elson's theorem [8] we obtain a unitary representation of $G$ on the completion of $X_{0}^{\lambda}$.

(2) If $m_{2 \alpha} \neq 0$ and $\lambda$ is real and $m_{2 \alpha}-1 \leqq \lambda<m_{\alpha}+m_{2 \alpha}+1,(,)_{\lambda}$ defines a positive definite Hermitian inner product on $X_{0}^{\lambda}$ and we have the same conclusion as above.

REMARKS (1) Theorems 3.1 and 3.2 show that the normalization given by Helgason [3] for the intertwining operators is the best possible one. (2) If one neglects the factor $c(i(\lambda-\rho))$ in the expression of $c(\lambda, \gamma)$, one obtains the quotient of two polynomials defined by Kostant [7]. 
4. Composition series and partial intertwining operators for the rank 1 case. Let $\operatorname{dim} \mathfrak{a}=1$. We have

Theorem 4.1 (1) (Kostant [7], Helgason [3]). $X_{0}^{\lambda}=X^{\lambda}$ if and only if $e(\lambda) \neq 0 . \pi_{\lambda}$ is irreducible if and only if $e(2 \rho-\lambda) e(\lambda) \neq 0$.

(2) (TAKAHASHI [11]). Let $m_{2 \alpha}=0$ and $\lambda=-k$ where $k$ is a nonnegative integer. Then $\pi_{-k}$ leaves $V_{k}=\sum_{j=0}^{k} X_{j}$ invariant, and $V_{k}$ and $X^{-k} / V_{k}$ are irreducible. $\pi_{m_{\alpha}+k}$ leaves $W_{k}=\sum_{j=k+1}^{\infty} X_{j}$ and $X^{m_{\alpha}+k}$ invariant, and $W_{k}$ and $X^{m_{\alpha}+k} / W_{k}$ are irreducible. As representations, $V_{k} \cong X^{m_{\alpha}+k} / W_{k}$ and $X^{-k} / V_{k} \cong W_{k}$. Furthermore, $\operatorname{Ker} A_{-k}=V_{k}$ and $A_{-k}$ induces a positive definite invariant inner product on $W_{k}$.

(3) Let $m_{2 \alpha}=1$ and let $\lambda=-2 l$ where $l$ is a nonnegative integer. Then $\pi_{-2 l}$ leaves $L_{2 l}=\sum_{m+|k| \leqq 2 l} X_{m, k}, H_{2 l}^{+}=\sum_{m-k \leqq 2 l} X_{m, k}$, $H_{2 l}^{-}=\sum_{m+k \leqq 2 l} X_{m, k}, H_{2 l}^{+}+H_{2 l}^{-}$and $X^{-2 m}$ invariant with $L_{2 l}, H_{2 l}^{+} / L_{2 l}$, $H_{2 l}^{-} / L_{2 l}$ and $X^{-2 l} /\left(H_{2 l}^{+}+H_{2 l}^{-}\right) \quad$ irreducible. $\pi_{m_{\alpha}+2+2 l}$ leaves $U_{2 l}$ $=\sum_{m+|k|>2 l} X_{m, k}, \quad F_{2 l}^{+}=\sum_{m+k>2 l} X_{m, k}, F_{2 l}^{-}=\sum_{m-k>2 l} X_{m, k}, F_{2 l}^{+}+F_{2 l}^{-}$ and $X^{m_{\alpha}+2+2 l}$ invariant with $U_{2 l}, F_{2 l}^{+} / U_{2 l}, F_{2 l}^{-} / U_{2 l}$, and $X^{m_{\alpha}+2+2 l} /\left(F_{2 l}^{-}+F_{2 l}^{+}\right)$ irreducible. As representations, $L_{2 l} \cong X^{m_{\alpha}+2+2 l} /\left(F_{2 l}^{-}+F_{2 l}^{+}\right), H_{2 l}^{+} / L_{2 l}$ $\cong F_{2 l}^{+} / U_{2 l}, H_{2 l}^{-} / L_{2 l} \cong F_{2 l}^{-} / U_{2 l}$, and $X^{-2 l} /\left(H_{2 l}^{+}+H_{2 l}^{-}\right) \cong U_{2 l} \cdot \operatorname{Ker} A_{-2 l}$ $=H_{2 l}^{+}+H_{2 l}^{-}$and, $A_{-2 l}$ induces an invariant positive definite inner product on $X^{-2 l} /\left(H_{2 l}^{+}+H_{2 l}^{-}\right)$. Setting $B_{\lambda}=\Gamma(\lambda / 2) A_{\lambda}$ then $B_{\lambda}$ is a meromorphic operator valued function and $B_{-2 l}\left|H_{2 l}^{+}, B_{-2 l}\right| H_{2 l}^{-}$induce nondegenerate invariant Hermitian products on $H_{2 l}^{+} / L_{2 l}$ and $H_{2 l}^{-} / L_{2 l}$.

These Hermitian products are positive definite if and only if $l=0$.

(4) (i) If $m_{2 \alpha}=3$ or 7 and $\lambda=-2 l$ with l a nonnegative integer we have that $W_{l}=\sum_{m+k \leqq 2 l} X_{m, k}, \quad M_{l}=\sum_{m-k \leqq 2 l+m_{2 \alpha}-1} X_{m, k}$, and $X^{-2 l}$ are invariant under $\pi_{-2 l}$ with $W_{l}, M_{l} / W_{l}$ and $X^{-2 l} / M_{l}$ are irreducible. $\tilde{W}_{l}$ $=\sum_{m+k>2 l} X_{m, k}, \tilde{M}_{l}=\sum_{m-k>2 l+m_{2 \alpha}-1} X_{m, k}$, and $X^{m_{\alpha}+2 m_{\alpha}+2 l}$ are invariant under $\pi_{m_{\alpha}+2 m_{2 \alpha}+2 l}$ with $\tilde{M}_{l}, \tilde{W}_{l} / \tilde{M}_{l}$ and $X^{m_{\alpha}+m_{2 \alpha}+2 l} / \tilde{W}_{l}$ irreducible. As representations $\quad \tilde{M}_{l} \cong X^{-2 l} / M_{l}, \quad \tilde{W}_{l} / \tilde{M}_{l} \cong M_{l} / W_{l}$ and $X^{m+2 m_{2}+2 l} / \tilde{W}_{l}$ $\cong W_{l}$. Ker $A_{-2 l}=M_{l}$ and $A_{-2 l}$ induces a positive definite inner product on $X^{-2 l} / M_{l}$.

(ii) Again, if $m_{2 \alpha}=3$ or 7 let $\lambda=2 l, l$ an integer $0<2 l \leqq m_{2 \alpha}-1$. Let $T_{l}=\sum_{m-k \leqq m_{2 x}-1-2 l} X_{m, k} . T_{l}$ is invariant and irreducible under $\pi_{2 l}$ and $X^{-2 l} / T_{l}$ is irreducible. $\widetilde{T}_{l}=\sum_{m-k>m_{2 \alpha}-1-2 l} X_{m, k}$ is invariant and irreducible under $\pi_{m_{\alpha}+2 m_{2 \alpha}-2 l}$ and $X^{m_{\alpha}+2 m_{2 \alpha}-2 l} / \widetilde{T}_{l}$ is irreducible. As representations $T_{l} \cong X^{m_{\alpha}+2 m_{2 \alpha}-2 l} / \widetilde{T}_{l}$ and $\widetilde{T}_{l} \cong X^{2 l} / T_{l}$. Furthermore, $T_{1}$ and all $\widetilde{T}_{l}$ possess positive definite invariant inner products.

Using partial intertwining operators as in (3), we obtain

THEOREM 4.2. Consider the representations of Theorem 4.1 which have positive definite invariant inner products. By Nelson's theorem [8], we obtain unitary representations of $G$ on their respective completions. 
5. Intertwining operators and generalized $c$-functions in the arbitrary rank case. Suppose now that $\operatorname{dim} \mathfrak{a}$ is arbitrary. Let $\Delta^{0}=\left\{\alpha \in \Delta \mid \frac{1}{2} \alpha \notin \Delta\right\}$, and if $w \in M^{\prime}$ let $\Delta^{0}(w)=\Delta^{0} \cap \Delta^{+} \cap w^{-1}\left(\Delta^{-}\right)$and $\bar{N}_{w}=\bar{N} \cap w^{-1} N w$. If $\alpha \in \Delta^{0}$ let $\mathfrak{g}^{\alpha}$ be the rank one algebra generated by $\mathfrak{g}_{-2 \alpha}, \mathfrak{g}_{-\alpha}, \mathfrak{g}_{\alpha}$ and $\mathfrak{g}_{2 \alpha}$. Let $G^{\alpha}$ denote the corresponding analytic subgroup of $G$. Let $K^{\alpha}=G^{\alpha} \cap K$, $\mathfrak{a}^{\alpha}=\mathfrak{g}^{\alpha} \cap \mathfrak{a}$ and $\alpha_{0}=\alpha /\langle\alpha, \alpha\rangle$.

Set

$$
d_{w}(\lambda)=\sum_{\alpha \in \Delta^{0}(w)} \Gamma\left(\left\langle\lambda, \alpha_{0}\right\rangle\right) 2^{-\left\langle\lambda, \alpha_{0}\right\rangle}
$$

and

$$
e_{w}(\lambda)^{-1}=\prod_{\alpha \in \Delta^{0}(w)} \Gamma\left(\frac{1}{2}\left(m_{\alpha}+1+\left\langle\lambda, \alpha_{0}\right\rangle\right)\right) \Gamma\left(\frac{1}{2}\left(m_{\alpha} / 2+m_{2 \alpha}+\left\langle\lambda, \alpha_{0}\right\rangle\right)\right) .
$$

For $w \in M^{\prime}, w=s_{p} \cdots s_{1}$ where each $s_{i}$ acts as a reflection on $\mathfrak{a}$. We say $l(w)=p$ if the above expression is of minimal length.

As $K$-representations $X^{\lambda}=X^{0}=\sum_{\gamma \in \hat{K}_{0}} n(\gamma) X_{\gamma}$ where $n(\gamma)=\operatorname{dim} X_{\gamma}^{M}$. Let $H_{\gamma}=n(\gamma) X_{\gamma}, \tau \in \gamma$, and $w \in M^{\prime}$ and consider the operator

$$
\begin{gathered}
T(\lambda, \gamma, w)=\frac{\tau(w)}{d_{w}(\lambda)} \int_{\bar{N}_{w}} \tau(k(\bar{n})) \exp -(\lambda+\rho)(H(\bar{n})) d \bar{n}, \\
T(\lambda, \gamma, w): H_{\gamma}^{M} \rightarrow H_{\gamma}^{M} .
\end{gathered}
$$

Let $B(\lambda, \gamma, w)$ denote the restriction of $T(\lambda, \gamma, w)$ to $H_{\gamma}^{M}$.

The results of Helgason [3] and Schiffmann [10] say that

(1) $\lambda \rightarrow B(\lambda, \gamma, w)$ is an analytic function; and,

(2) if $w, w^{\prime}, w^{\prime \prime} \in M^{\prime}$ with $w=w^{\prime} w^{\prime \prime}$ and $l(w)=l\left(w^{\prime}\right)+l\left(w^{\prime \prime}\right), B(\lambda, \gamma, w)$ $=B\left(w^{\prime \prime}(\lambda), \gamma, w^{\prime}\right) B\left(\lambda, \gamma, w^{\prime \prime}\right)$.

THEOREM 5.1. (1) $B(\lambda, \gamma, w)$ is an isomorphism for all $\gamma \in \hat{K}_{0}$ if and only if $e_{w}(\lambda) \neq 0$. Moreover, $\pi_{\lambda+\rho}$ and $\pi_{w \lambda+\rho}$ are equivalent.

(2) There is $a \gamma \in \hat{K}_{0}$ such that $B(\lambda, \gamma, w)$ is not identically 0 .

(3) Let $s_{\alpha}$ be the reflection of or about the hyperplane $\alpha=0$. Let $\gamma \in \hat{K}_{0}$. Then $V_{\gamma}=\sum_{\beta \in \hat{K}_{0}^{\alpha}} m_{\alpha}(\gamma, \beta) V_{\beta}$ as a $K^{\alpha}$ representation where $m_{\alpha}(\gamma, \beta)$ denotes the multiplicity of $V_{\beta}$ in $V_{\gamma}$.

Moreover,

$$
\begin{gathered}
V_{\gamma}^{M}=\sum_{\beta \in \hat{R}_{0}^{\alpha}} m_{\alpha}(\gamma, \beta) V_{\beta}^{M^{\alpha}}, \\
\left.B\left(\lambda, \gamma, s_{\alpha}\right)\right|_{m_{x}(\gamma, \beta) V M_{\beta}^{\alpha}}=a\left(\left.\alpha(\lambda+\rho)\right|_{a} \beta\right) I .
\end{gathered}
$$

Notes. (1) (ii) says that $B\left(\lambda, \gamma, s_{\alpha}\right)$ diagonalizes according to the spliiting of $V_{\gamma}$ as a representation of $K^{\alpha}$.

(2) $\prod_{\alpha \in \Delta^{0}}$ det $B\left(\lambda, \gamma, s_{\alpha}\right)$ is up to constant factor independent of $\lambda$, $\boldsymbol{P}_{\gamma}(2 \rho-\lambda) / \boldsymbol{P}_{\gamma}(\lambda)$, where $\boldsymbol{P}_{\gamma}(\lambda)$ is the polynomial defined in Kostant [7]. 


\section{BIBLIOGRAPHY}

1. I. M. Gel'fand, M. I. Graev and N. Ja. Vilenkin, Generalized functions. Vol. 5: Integral geometry and representation theory, Fizmatgiz, Moscow, 1962; English transl., Academic Press, New York, 1966. MR 28 \# 3324; MR 34 \# 7726.

2. Harish-Chandra, Representations of semisimple Lie groups. II, Trans. Amer. Math. Soc. 76 (1954), 26-65. MR 15, 398.

3. S. Helgason, $A$ duality for symmetric spaces with applications to group representations, Advances in Math. 5 (1970), 1-154. MR 41 \# 8587.

4. K. Johnson, Composition series and intertwining operators for the spherical principal series. II (to appear).

5. K. Johnson and N. Wallach, Composition series and intertwining operators for the spherical principal series. I (to appear).

6. A. Knapp and E. M. Stein, Intertwining operators for semisimple groups, Ann. of Math. (2) 93 (1971), 489-578.

7. B. Kostant, On the existence and irreducibility of certain series of representations, Bull. Amer. Math. Soc. 75 (1969), 627-642. MR 39 \# 7031.

8. E. Nelson, Analytic vectors, Ann. of Math. (2) 70 (1959), 572-615. MR 21 \# 5901.

9. P. Sally, Intertwining operators and the representations of $\operatorname{SL}(2, \boldsymbol{R})$, J. Functional Analysis 6 (1970), 441-453.

10. G. Schiffmann, Intégrales d'entrelacement et fonctions de Whittaker, Bull. Soc. Math. France 99 (1971), 3-72.

11. R. Takahashi, Sur les représentations unitaires des groupes de Lorentz généralisés, Bull. Soc. Math. France 91 (1963), 289-433. MR 31 \# 3544.

Department of Mathematics, Rutgers University, New Brunswick, New Jersey 08903

Current address (Kenneth Johnson): Department of Mathematics, Rice University, Houston, Texas 77001 